\title{
Ichthyofauna of Mundaú river basin, Ceará State, Northeastern Brazil
}

\author{
Francisco Keilo Teixeira ${ }^{1}$, Telton Pedro Anselmo Ramos ${ }^{2,3}$, Roney Emanuel Costa de Paiva ${ }^{2}$, \\ Marcelo Aguiar Távora ${ }^{1,4}$, Sergio Maia Queiroz Lima² \& Carla Ferreira Rezende ${ }^{I}$ \\ ${ }^{1}$ Universidade Federal do Ceará, Departamento de Biologia, Fortaleza, CE, Brazil \\ ${ }^{2}$ Universidade Federal do Rio Grande do Norte, Departamento de Botânica e Zoologia, Natal, RN, Brazil \\ ${ }^{3}$ Universidade Federal da Paraíba, Centro de Ciências Exatas e da Natureza, Departamento de Sistemática e \\ Ecologia, João Pessoa, PB, Brazil \\ ${ }^{4}$ Instituto Federal de Educação, Ciência e Tecnologia do Rio Grande do Norte, Ipanguaçu, RN, Brazil \\ *Corresponding author:Telton Pedro Anselmo Ramos,e-mail:telton@gmail.com
}

TEIXEIRA, F.K., RAMOS, T.P.A., PAIVA, R.E.C., TÁVORA, M.A., LIMA, S.M.Q., REZENDE, C.F. Ichthyofauna of Mundaú river basin, Ceará State, Northeastern Brazil. Biota Neotropica. 17(1): e20160174. http://dx.doi. org/10.1590/1676-0611-BN-2016-0174

\begin{abstract}
Mundaú river basin is located at Center-North Ceará State and occupies a total area of 2,227 $\mathrm{km}^{2}$, including Estuário do Rio Mundaú Environmental Protection Area. This study aimed to catalog the fishes of this basin. Collections were performed with active and passive gear in 35 sampling sites, between 2012 and 2014, in several habitats (main channels, streams, floodplains, permanent and temporary pools, ponds, and dams). A total of 2,545 specimens were collected, belonging to 55 species distributed in 10 orders, 31 families, and 50 genera; 30 of these are strictly freshwater species, and 25 estuarine-marine species. Three species (Hemigrammus guyanensis Gery, 1995, H. rodwayi Durbin, 1909 and Poecilia sarrafae Bragança \& Costa, 2011) represent new records for the Mid-Northeastern Caatinga ecoregion. Besides, two cynolebiid species, Hypsolebias sp. and Anablepsoides cearensis (Costa \& Vono, 2009), were found and the latter, currently classified as critically endangered, had its occurrence area widened.
\end{abstract}

Keywords: intermittent river, semiarid, Mid-Northeastern ecoregion, freshwater neotropical fishes.

\section{Ictiofauna da bacia do rio Mundaú, Estado do Ceará, Nordeste do Brasil}

\begin{abstract}
Resumo: A bacia do Rio Mundaú está situada no centro-norte do Estado do Ceará, e drena uma área de $2.227 \mathrm{~km}^{2}$ de Caatinga, incluindo a Área de Proteção Ambiental do Estuário do Rio Mundaú. Este estudo teve como objetivo realizar um inventário dos peixes desta bacia. As coletas foram realizadas com petrechos ativos e passivos, em 35 pontos amostrais entre 2012 e 2014 em diversos habitats (canal principal, córregos, alagados, poças permanentes e temporárias, lagoas e açudes) ao longo de toda a bacia. Um total de 2.545 indivíduos foram coletados, pertencentes a 55 espécies distribuídas em 10 ordens, 31 famílias e 50 gêneros; destas, 30 são estritamente de água doce e 25 estuarino-marinhas. Três espécies (Hemigrammus guyanensis Gery,1995, H. rodwayi Durbin, 1909 e Poecilia sarrafae Bragança \& Costa, 2011) constituem novas ocorrências para a ecorregião do Nordeste Médio-Oriental. Além disso, foram registradas duas espécies de cinolebídeos, Hypsolebias sp. e Anablepsoides cearensis (Costa \& Vono, 2009), a última classificada como criticamente ameaçada de extinção, teve sua área de ocorrência ampliada.
\end{abstract}

Palavras-chave: rios intermitentes, semiárido, ecorregião Nordeste Médio-Oriental, peixes de água doce neotropical.

\section{Introduction}

Freshwater ichthyofaunal studies in Brazil are mainly focused on South and Southeastern regions (Langeani et al. 2009). While these regions are well documented, the Northeastern region is in need of studies, especially the Mid-Northeastern Caatinga Ecoregion (MNCE) (Rosa et al. 2003; Ramos et al. 2005). This ecoregion includes coastal basins located between São Francisco and Parnaíba river basins, draining Alagoas, Pernambuco, Paraíba, Rio Grande do Norte, Ceará, and a small portion of Piauí States (Rosa et al. 2003). Albert et al. (2011) listed 88 freshwater fish species from MNCE; however, new species are being found and described (e. g. Parotocinclus seridoensis Ramos, Barro-Neto, Britski \& Lima, 2013; Serrapinnus potiguar Jerep \& Malabarba, 2014; and Hypsolebias martinsi Britzke, Nielsen \& Oliveira, 2016). The estimative of freshwater fish species number at Brazilian Northeast may be premature, given the lack of taxonomic revisions and few representative regional collections (Rosa et al. 2003; Langeani et al. 2009, Ramos et al. 2014).

Ichthyofaunistic inventories at MNCE basins are scarce (Langeani et al. 2009), and the existing ones usually deal with small portions of bigger basins (e.g. Silva et al. 2014), or reservoirs (e.g. Gurgel-Lourenço et al. 2013, Sánchez-Botero et al. 2014). Few coastal basins in this hydrographical region were fully cataloged, for example Gramame river basin, in 
Paraíba State, in which (Gomes-Filho \& Rosa 2001) there were recorded 23 species; Curimataú river basin in Paraíba and Rio Grande do Norte States, 22 species (Ramos et al. 2005), and Pratagi river microbasin, also in Rio Grande do Norte State, 22 species (Paiva et al. 2014).

MNCE is largely inserted in the Brazilian semiarid, an area of Caatinga's phytophysionomy where rivers are mostly intermittent resulting in a simple hydrographical network (Rosa et al. 2003), except for headwaters and coastal areas in Alagoas, Pernambuco, Paraíba, and Rio Grande do Norte States, which are greatly nested within the Atlantic Forest (Rosa \& Groth 2004, Paiva et al. 2014). Therefore, these semiarid river systems are constantly being modified through their damming as an attempt to assure the inland population a hydric demand during long drought periods.

Except Jaguaribe river basin and a small portion of Parnaíba river basin, Ceará hydrographic network is composed by small-sized coastal basins. Some of these basins may probably have an important role to the conservation of Caatinga's fishes, although they have been considered insufficiently known (MMA 2007). Among these, there is Mundaú river basin, located in Center-North Ceará State, whose freshwater ichthyofauna is virtually unknown. Soares-Filho et al. (2010) cataloged the estuarine porcion of this basin and reported 53 estuarine and marine fish species. The Estuário do Rio Mundaú Environmental Protection Area (EPA) is located at the lower extent of the basin, covering 1,596.37 hectares of Ceará West coast, and it is considered a priority area for fish conservation (SEMACE 2014). Because of the basin's importance for the Caatinga fish fauna knowledge, this study aimed to survey the fishes of Mundaú river basin.

\section{Material and methods}

\section{Study area}

Mundaú river basin (Figure 1) covers, approximately, an area of $2,227 \mathrm{~km}^{2}$ (COGERH 2014), draining regional residual massifs, backland depressions, and seaside plains (IPECE 2012). The two main tributaries of Mundaú river basin, Cruxati in the west and Mundaú in the east, emerge from Uruburetama Massif at elevations up to $970 \mathrm{~m}$, and are intermittent. Along their courses, these rivers are fed by various first order waterbodies, like Sororô, Tabocas, Laginhas, and Torrados streams. Mundaú river main course runs $97.6 \mathrm{~km}$ north to south, and disembogues in the Atlantic Ocean, while Cruxati river runs $77.5 \mathrm{~km}$ from headwaters to Mundaú river's confluence (COGERH 2014).

The local climate is semiarid with mean annual precipitation of $1,110.6 \mathrm{~mm}$, and $1,914.7 \mathrm{~mm}$ evaporation; mean annual temperatures between $26^{\circ} \mathrm{C}$ and $28^{\circ} \mathrm{C}$, and highest precipitations occurring from January to June (FUNCEME 2015).

\subsection{Sampling design}

Acquisition of specimens was performed in 35 locations along the entire Mundaú river basin. For better coverage, collections were made at Cruxati and Mundaú rivers' main channels, as well as in streams, ponds, dams, and bogs (Table 1). Sampling took place during dry and wet seasons, between 2012 and 2014, covering localities from Mundaú river headwaters, $900 \mathrm{~m}$

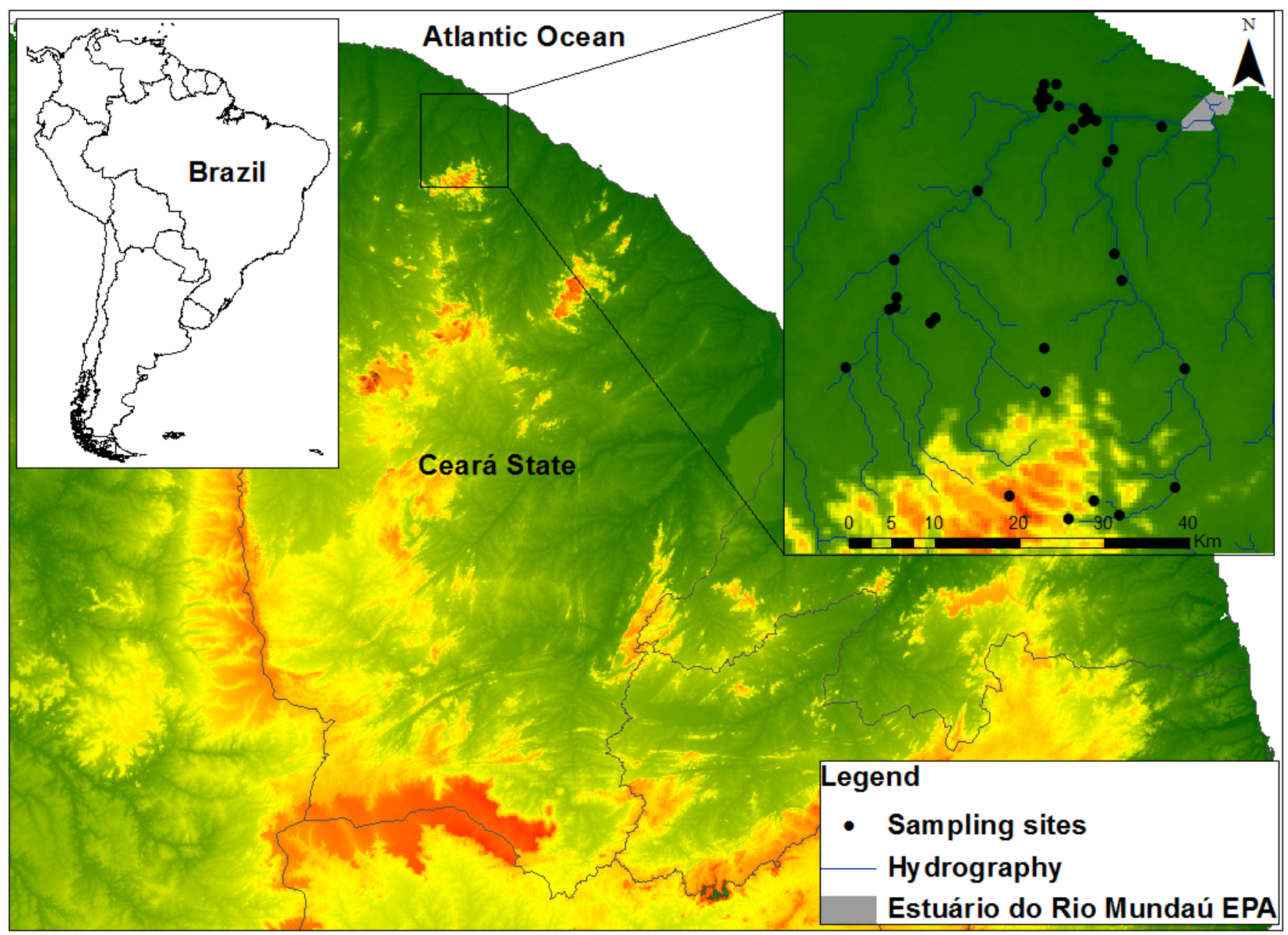

Figure 1. Mundaú river basin location in Ceará State, northeastern Brazil, evidencing the sampling sites. Hot colors represent higher altitudes. 
Ichthyofauna of Mundaú River basis

Table 1. Geographic coordinates, elevation, tributary and main habitat of the sampling sites along the Mundáu river basin.

\begin{tabular}{|c|c|c|c|}
\hline Geographic Coordinates & Elevation (m) & Tributary & Habitat \\
\hline $3.62527^{\circ} \mathrm{S} 39.49782^{\circ} \mathrm{W}$ & 108 & Mundaú & Main channel \\
\hline $3.21225^{\circ} \mathrm{S} 39.45283^{\circ} \mathrm{W}$ & 11 & Mundaú & Estuary \\
\hline $3.46911^{\circ} \mathrm{S} 39.42811^{\circ} \mathrm{W}$ & 51 & Mundaú & Main channel \\
\hline $3.59491^{\circ} \mathrm{S} 39.43911^{\circ} \mathrm{W}$ & 89 & Mundaú & Main channel \\
\hline $3.60494^{\circ} \mathrm{S} 39.61399^{\circ} \mathrm{W}$ & 913 & Mundaú & Headwater \\
\hline $3.62936^{\circ} \mathrm{S} 39.55111^{\circ} \mathrm{W}$ & 273 & Mundaú & Main channel \\
\hline $3.46814^{\circ} \mathrm{S} 39.78777^{\circ} \mathrm{W}$ & 56 & Cruxati & Stream \\
\hline $3.44799^{\circ} \mathrm{S} 39.57688^{\circ} \mathrm{W}$ & 80 & Cruxati & Pond \\
\hline $3.42094^{\circ} \mathrm{S} 39.69766^{\circ} \mathrm{W}$ & 62 & Cruxati & Stream \\
\hline $3.41619^{\circ} \mathrm{S} 39.69288^{\circ} \mathrm{W}$ & 70 & Cruxati & Rock pool \\
\hline $3.40675^{\circ} \mathrm{S} 39.74111^{\circ} \mathrm{W}$ & 44 & Cruxati & Main channel \\
\hline $3.40438^{\circ} \mathrm{S} 39.73577^{\circ} \mathrm{W}$ & 43 & Cruxati & Stream \\
\hline $3.39431^{\circ} \mathrm{S} 39.73433^{\circ} \mathrm{W}$ & 41 & Cruxati & Main channel \\
\hline $3.37569^{\circ} \mathrm{S} 39.49455^{\circ} \mathrm{W}$ & 34 & Mundaú & Dam \\
\hline $3.61006^{\circ} \mathrm{S} 39.52488^{\circ} \mathrm{W}$ & 145 & Mundaú & Headwater stream \\
\hline $3.35383^{\circ} \mathrm{S} 39.73588^{\circ} \mathrm{W}$ & 37 & Cruxati & Pool on the main channel \\
\hline $3.34763^{\circ} \mathrm{S} 39.50277^{\circ} \mathrm{W}$ & 23 & Mundaú & Pool on the main channel \\
\hline $3.28102^{\circ} \mathrm{S} 39.64833^{\circ} \mathrm{W}$ & 17 & Cruxati & Pool on the main channel \\
\hline $3.24950^{\circ} \mathrm{S} 39.51011^{\circ} \mathrm{W}$ & 6 & Mundaú & Estuary \\
\hline $3.23722^{\circ} \mathrm{S} 39.50433^{\circ} \mathrm{W}$ & 8 & Mundaú & Estuary \\
\hline $3.21582^{\circ} \mathrm{S} 39.54688^{\circ} \mathrm{W}$ & 10 & Mundaú & Floodplain \\
\hline $3.20777^{\circ} \mathrm{S} 39.53649^{\circ} \mathrm{W}$ & 9 & Mundaú & Floodplain \\
\hline $3.20650^{\circ} \mathrm{S} 39.52169^{\circ} \mathrm{W}$ & 8 & Mundaú & Estuary \\
\hline $3.20456^{\circ} \mathrm{S} 39.53277^{\circ} \mathrm{W}$ & 9 & Cruxati & Dam \\
\hline $3.19706^{\circ} \mathrm{S} 39.53098^{\circ} \mathrm{W}$ & 9 & Mundaú & Estuary \\
\hline $3.19319^{\circ} \mathrm{S} 39.53528^{\circ} \mathrm{W}$ & 8 & Cruxati & Floodplain \\
\hline $3.19197^{\circ} \mathrm{S} 39.57933^{\circ} \mathrm{W}$ & 13 & Cruxati & Estuary \\
\hline $3.19114^{\circ} \mathrm{S} 39.56165^{\circ} \mathrm{W}$ & 7 & Mundaú & Pool on the main channel \\
\hline $3.18395^{\circ} \mathrm{S} 39.58353^{\circ} \mathrm{W}$ & 21 & Cruxati & Stream \\
\hline $3.18297^{\circ} \mathrm{S} 39.57402^{\circ} \mathrm{W}$ & 14 & Cruxati & Pond \\
\hline $3.17528^{\circ} \mathrm{S} 39.57939^{\circ} \mathrm{W}$ & 33 & Cruxati & Stream \\
\hline $3.16827^{\circ} \mathrm{S} 39.56391^{\circ} \mathrm{W}$ & 29 & Cruxati & Floodplains \\
\hline $3.16748^{\circ} \mathrm{S} 39.57683^{\circ} \mathrm{W}$ & 26 & Cruxati & Floodplains \\
\hline $3.49422^{\circ} \mathrm{S} 39.57544^{\circ} \mathrm{W}$ & 102 & Cruxati & Stream \\
\hline $3.20361^{\circ} \mathrm{S} 39.52817^{\circ} \mathrm{W}$ & 7 & Mundaú & Estuary \\
\hline
\end{tabular}

higher than the estuary. Due to river intermittence, dry season samples were taken only in remaining pools at the main watercourse, while wet season sampling covered locations in the main flow and marginal environments.

Fish were captured using seines (seine nets $20 \times 2.5 \mathrm{~m}$, mesh size $10 \mathrm{~mm}$ and $4 \times 2 \mathrm{~m}$, mesh size $5 \mathrm{~mm}$ ), castnets ( $2 \mathrm{~m}$ height, mesh size $15 \mathrm{~mm}$ ), gillnets of various mesh sizes $(15,25,35,45$ and $55 \mathrm{~mm})$, and dip nets (mesh size 5 and $10 \mathrm{~mm}$ ) and bottle traps. Fishes were anesthetized with $30 \mathrm{~mL}$ of a $10 \%$ clove oil solution (clove oil $10 \mathrm{~mL}$; ethyl alcohol $90 \mathrm{~mL}$ ) in $970 \mathrm{~mL}$ of water (Lucena et al. 2013), fixed in a 4\% formaldehyde solution during a minimal eight-day period, and conserved in $70 \%$ ethanol solution. Fish surveys were conducted according to governmental laws (Permit $\mathrm{n}^{\circ}$ 17632-2/ICMBio). Fishes were screened, identified, and deposited at the ichthyological collections of Universidade Federal do Rio Grande do Norte (UFRN) and Universidade Federal da Paraíba (UFPB). The fishes were identified to the lowest taxonomic level according to specialized sources, that is, group specific identification keys, systematic reviews, original descriptions (e.g. Araújo et al. 2004, Britski et al. 1984, Costa 2007, Marceniuk 2005, Kullander 1988, Ploeg 1991, Ramos 2012), and specialist support. Species classified as new occurrences were evaluated according to Reis et al. (2003), Rosa et al. (2003), and Buckup et al. (2007).
The nomenclature followed the recommendation by Eschmeyer (2015), and species habitat definition followed Fishbase (2014).

\section{Results}

A total of 2,545 fish specimens were collected, belonging to 55 species, 50 genera, 31 families, and 10 orders (Table 2). Of those species, $30(54.5 \%)$ are strictly freshwater, seven (12.7\%) estuarine, and $14(25.4 \%)$ marine (Table 2). Astyanax aff. bimaculatus (Linnaeus, 1758) was widely distributed, being present in 19 of 35 sampling sites. Poecilia vivipara Bloch \& Schneider, 1801, Awaous tajasica (Lichtenstein, 1822), and Dormitator maculatus (Bloch, 1792) were the only species present in both fresh Bragança \& Costa, 2011 and brackish waters. Oreochromis niloticus (Linnaeus, 1758) and Poecilia reticulata Peters, 1859 are exotic introduced species. Poecilia sarrafae, Hemigrammus guyanensis Géry, 1959, and H. rodwayi Durbin, 1909 are new occurrences for MNCE, and Nannostomus beckfordi Günther, 1872 and Callichthys callichthys (Linnaeus, 1758) are new records for Ceará State.

Among freshwater fishes, Characiformes was the most representative order in number of species (53.3\%), while among marine and estuarine fishes it was Perciformes (80\%). The orders Siluriformes and Cyprinodontiformes represent $10.9 \%$ and $9.1 \%$ of total species, with six and five species, 
Table 2. List of fish species and information on physiology, habitat, abundance and voucher of the Mundaú river basin. Abbreviations: ${ }^{(\mathrm{E})}$ Estuarine, ${ }^{(\mathrm{F})}$ freshwater; ${ }^{(\mathrm{M})}$ marine; (NR) new record for the MNCE; (CR) Critically endangered; ${ }^{(I)}$ introduced; ${ }^{(*)}$ Photographed voucher.

\begin{tabular}{|c|c|c|c|c|c|c|c|c|c|}
\hline ORDER/Family/Species & FISIOLOGY & $\begin{array}{c}\text { MAIN } \\
\text { CHANNEL } \\
\end{array}$ & STREAM & $\begin{array}{l}\text { FLOOD } \\
\text { PLAIN }\end{array}$ & $\begin{array}{l}\text { ROCK } \\
\text { POOL }\end{array}$ & POND & DAM & $\begin{array}{c}\text { TOTAL } \\
\text { ABUNDANCE } \\
\end{array}$ & VOUCHER \\
\hline \multicolumn{10}{|l|}{ ELOPIFORMES (1) } \\
\hline \multicolumn{10}{|l|}{ Elopidae (1) } \\
\hline Elops saurus Linnaeus, 1766 & M & 6 & 0 & 0 & 0 & 0 & 0 & 6 & UFRN 2612 \\
\hline \multicolumn{10}{|l|}{ CHARACIFORMES (16) } \\
\hline \multicolumn{10}{|l|}{ Curimatidae (1) } \\
\hline $\begin{array}{l}\text { Steindachnerina notonota } \\
\text { (Miranda Ribeiro, 1937) }\end{array}$ & $\mathrm{F}$ & 122 & 22 & 20 & 0 & 8 & 25 & 197 & UFRN 2642 \\
\hline \multicolumn{10}{|l|}{ Prochilodontidae (1) } \\
\hline $\begin{array}{l}\text { Prochilodus brevis Steindachner, } \\
1875\end{array}$ & $\mathrm{~F}$ & 42 & 8 & 0 & 5 & 15 & 29 & 99 & UFRN 3530 \\
\hline \multicolumn{10}{|l|}{ Anostomidae (1) } \\
\hline Leporinus piau Fowler, 1941 & $\mathrm{~F}$ & 7 & 15 & 0 & 0 & 0 & 0 & 22 & UFRN 1421 \\
\hline \multicolumn{10}{|l|}{ Erythrinidae (1) } \\
\hline $\begin{array}{l}\text { Hoplias malabaricus } \\
\text { (Bloch, 1794) }\end{array}$ & $\mathrm{F}$ & 14 & 4 & 2 & 0 & 3 & 4 & 27 & UFRN 2582 \\
\hline \multicolumn{10}{|l|}{ Lebiasinidae (1) } \\
\hline $\begin{array}{l}\text { Nannostomus beckfordi Günther, } \\
1872^{\text {(NR) }}\end{array}$ & $\mathrm{F}$ & 24 & 19 & 15 & 0 & 3 & 44 & 105 & UFRN 2591 \\
\hline \multicolumn{10}{|l|}{ Characidae (10) } \\
\hline $\begin{array}{l}\text { Astyanax aff. bimaculatus } \\
\text { (Linnaeus 1758) }\end{array}$ & $\mathrm{F}$ & 204 & 36 & 4 & 23 & 8 & 24 & 299 & UFRN 1420 \\
\hline $\begin{array}{l}\text { Astyanax aff. fasciatus } \\
\text { (Cuvier 1819) }\end{array}$ & $\mathrm{F}$ & 114 & 15 & 0 & 0 & 20 & 0 & 149 & UFRN 1432 \\
\hline $\begin{array}{l}\text { Cheirodon jaguaribensis } \\
\text { Fowler,1941 }\end{array}$ & $\mathrm{F}$ & 5 & 0 & 0 & 0 & 0 & 0 & 5 & UFRN 2523 \\
\hline $\begin{array}{l}\text { Compsura heterura } \\
\text { (Eigenmann, 1915) }\end{array}$ & $\mathrm{F}$ & 101 & 12 & 11 & 0 & 8 & 0 & 132 & UFRN 1430 \\
\hline $\begin{array}{l}\text { Hemigrammus guyanensis Géry, } \\
1959^{\text {(NR) }}\end{array}$ & $\mathrm{F}$ & 5 & 6 & 1 & 0 & 0 & 0 & 12 & UFRN 2599 \\
\hline $\begin{array}{l}\text { Hemigrammus rodwayi Durbin, } \\
1909^{\text {(NR) }}\end{array}$ & $\mathrm{F}$ & 41 & 30 & 4 & 0 & 3 & 0 & 78 & UFRN 2562 \\
\hline Hyphessobrycon sp. & $\mathrm{F}$ & 0 & 32 & 0 & 0 & 0 & 4 & 36 & UFRN 2602 \\
\hline $\begin{array}{l}\text { Phenacogaster calverti } \\
\text { (Fowler, 1941) }\end{array}$ & $\mathrm{F}$ & 114 & 8 & 12 & 0 & 8 & 11 & 153 & UFRN 2557 \\
\hline $\begin{array}{l}\text { Serrapinnus heterodon } \\
\text { (Eigenmann 1915) }\end{array}$ & $\mathrm{F}$ & 187 & 39 & 11 & 12 & 17 & 22 & 288 & UFRN 1431 \\
\hline $\begin{array}{l}\text { Serrapinnus piaba } \\
\text { (Lütken, 1875) }\end{array}$ & $\mathrm{F}$ & 132 & 18 & 11 & 7 & 12 & 11 & 191 & UFRN 1429 \\
\hline \multicolumn{10}{|l|}{ Crenuchidae (1) } \\
\hline $\begin{array}{l}\text { Characidium bimaculatum } \\
\text { Fowler, } 1941\end{array}$ & $\mathrm{~F}$ & 10 & 5 & 0 & 0 & 0 & 0 & 15 & UFRN 1439 \\
\hline \multicolumn{10}{|l|}{ SILURIFORMES (6) } \\
\hline \multicolumn{10}{|l|}{ Ariidae (2) } \\
\hline Sciades herzbergii (Bloch, 1794) & M & 11 & 0 & 0 & 0 & 0 & 0 & 11 & UFRN 3550 \\
\hline \multicolumn{10}{|l|}{ Auchenipteridae (1) } \\
\hline $\begin{array}{l}\text { Trachelyopterus galeatus } \\
\text { (Linnaeus, 1766) }\end{array}$ & $\mathrm{F}$ & 4 & 0 & 0 & 0 & 0 & 0 & 4 & UFRN 3531 \\
\hline \multicolumn{10}{|l|}{ Callichthyidae (1) } \\
\hline $\begin{array}{l}\text { Callichthys callichthys } \\
\text { (Linnaeus, 1758) }\end{array}$ & $\mathrm{F}$ & 1 & 1 & 0 & 0 & 0 & 0 & 2 & UFRN 2607 \\
\hline \multicolumn{10}{|l|}{ Loricariidae (2) } \\
\hline $\begin{array}{l}\text { Hypostomus cf. pusarum } \\
\text { (Starks, 1913) }\end{array}$ & $\mathrm{F}$ & 9 & 0 & 0 & 0 & 0 & 4 & 13 & UFRN 2584 \\
\hline $\begin{array}{l}\text { Parotocinclus cearensis } \\
\text { Garavello, } 1977\end{array}$ & F & 4 & 0 & 0 & 0 & 0 & 0 & 4 & UFRN 1422 \\
\hline
\end{tabular}


Table 2. Continued...

\begin{tabular}{|c|c|c|c|c|c|c|c|c|c|}
\hline ORDER/Family/Species & FISIOLOGY & $\begin{array}{c}\text { MAIN } \\
\text { CHANNEL }\end{array}$ & STREAM & $\begin{array}{l}\text { FLOOD } \\
\text { PLAIN }\end{array}$ & $\begin{array}{l}\text { ROCK } \\
\text { POOL }\end{array}$ & POND & DAM & $\begin{array}{c}\text { TOTAL } \\
\text { ABUNDANCE }\end{array}$ & VOUCHER \\
\hline \multicolumn{10}{|l|}{ BATRACHOIDIFORMES (1) } \\
\hline \multicolumn{10}{|l|}{ Batrachoididae (1) } \\
\hline $\begin{array}{l}\text { Batrachoides surinamensis } \\
\text { (Bloch \& Schneider, 1801) }\end{array}$ & M & 1 & 0 & 0 & 0 & 0 & 0 & 1 & UFRN 3545 \\
\hline \multicolumn{10}{|l|}{ MUGILIFORMES (1) } \\
\hline \multicolumn{10}{|l|}{ Mugilidae (1) } \\
\hline $\begin{array}{l}\text { Mugil curema Velenciennes, } \\
1836\end{array}$ & M & 24 & 0 & 0 & 0 & 0 & 0 & 24 & UFRN 2611 \\
\hline \multicolumn{10}{|l|}{ CYPRINODONTIFORMES (5) } \\
\hline \multicolumn{10}{|l|}{ Cynolebiidae (2) } \\
\hline $\begin{array}{l}\text { Anablepsoides cearensis (Costa } \\
\& \text { Vono, 2009) }\end{array}$ & $\mathrm{F}$ & 0 & 9 & 20 & 0 & 0 & 0 & 29 & $\begin{array}{l}\text { UFRN } \\
3046^{*}\end{array}$ \\
\hline $\begin{array}{l}\text { Hypsolebias sp. } \\
\text { Poeciliidae (3) }\end{array}$ & $\mathrm{F}$ & 8 & 12 & 8 & 0 & 0 & 0 & 28 & UFRN 1446 \\
\hline Poecilia reticulata Peters, $1859^{(1)}$ & $\mathrm{F}$ & 36 & 31 & 0 & 0 & 0 & 0 & 67 & UFRN 2581 \\
\hline $\begin{array}{l}\text { Poecilia sarrafae Bragança \& } \\
\text { Costa, 2011 }\end{array}$ & $\mathrm{F}$ & 0 & 20 & 26 & 0 & 0 & 0 & 46 & UFRN 2574 \\
\hline $\begin{array}{l}\text { Poecilia vivipara Bloch \& } \\
\text { Schneider, } 1801\end{array}$ & $\mathrm{~F}$ & 113 & 28 & 10 & 29 & 12 & 6 & 198 & UFRN 1419 \\
\hline \multicolumn{10}{|l|}{ SYNBRANCHIFORMES (1) } \\
\hline \multicolumn{10}{|l|}{ Synbranchidae (1) } \\
\hline $\begin{array}{l}\text { Synbranchus marmoratus Bloch, } \\
1795\end{array}$ & $\mathrm{~F}$ & 2 & 0 & 0 & 0 & 0 & 0 & 2 & UFRN 3534 \\
\hline \multicolumn{10}{|l|}{ PERCIFORMES (20) } \\
\hline \multicolumn{10}{|l|}{ Centropomidae (1) } \\
\hline $\begin{array}{l}\text { Centropomus undecimalis } \\
\text { (Bloch, 1792) }\end{array}$ & M & 5 & 0 & 0 & 0 & 0 & 0 & 5 & UFRN 3546 \\
\hline \multicolumn{10}{|l|}{ Carangidae (2) } \\
\hline Hemicaranx sp. & M & 4 & 0 & 0 & 0 & 0 & 0 & 4 & UFRN 3541 \\
\hline $\begin{array}{l}\text { Oligoplites saurus } \\
\text { (Bloch \& Schneider, 1801) }\end{array}$ & M & 4 & 0 & 0 & 0 & 0 & 0 & 4 & UFRN 3548 \\
\hline \multicolumn{10}{|l|}{ Lutjanidae (1) } \\
\hline $\begin{array}{l}\text { Lutjanus sp. } \\
\text { Gerreidae (3) }\end{array}$ & M & 3 & 0 & 0 & 0 & 0 & 0 & 3 & UFRN 3538 \\
\hline $\begin{array}{l}\text { Eugerres brasilianus (Cuvier, } \\
1830 \text { ) }\end{array}$ & M & 4 & 0 & 0 & 0 & 0 & 0 & 4 & UFRN 3549 \\
\hline Diapterus auratus Ranzani,1842 & M & 23 & 0 & 0 & 0 & 0 & 0 & 23 & UFRN 3655 \\
\hline $\begin{array}{l}\text { Eucinostomus argenteus Baird } \\
\text { \& Girard, } 1855\end{array}$ & M & 8 & 0 & 0 & 0 & 0 & 0 & 8 & UFRN 3654 \\
\hline \multicolumn{10}{|l|}{ Haemulidae (2) } \\
\hline $\begin{array}{l}\text { Genyatremus luteus } \\
\text { (Bloch, 1970) }\end{array}$ & M & 5 & 0 & 0 & 0 & 0 & 0 & 5 & UFRN 3535 \\
\hline $\begin{array}{l}\text { Haemulopsis corvinaeformis } \\
\text { (Steindachner 1868) }\end{array}$ & M & 8 & 0 & 0 & 0 & 0 & 0 & 8 & UFRN 3542 \\
\hline \multicolumn{10}{|l|}{ Sparidae (1) } \\
\hline $\begin{array}{l}\text { Archosargus probatocephalus } \\
\text { (Walbaum, 1792) }\end{array}$ & $\mathrm{E}$ & 10 & 0 & 0 & 0 & 0 & 0 & 10 & UFRN 4281 \\
\hline \multicolumn{10}{|l|}{ Sciaenidae (2) } \\
\hline $\begin{array}{l}\text { Bairdiella ronchus (Cuvier, } \\
\text { 1830) }\end{array}$ & M & 5 & 0 & 0 & 0 & 0 & 0 & 5 & UFRN 3537 \\
\hline Larimus breviceps Cuvier, 1830 & M & 11 & 0 & 0 & 0 & 0 & 0 & 11 & UFRN 3540 \\
\hline \multicolumn{10}{|l|}{ Ephippidae (1) } \\
\hline $\begin{array}{l}\text { Chaetodipterus faber } \\
\text { (Broussonet, 1782) }\end{array}$ & M & 15 & 0 & 0 & 0 & 0 & 0 & 15 & UFRN 3547 \\
\hline \multicolumn{10}{|l|}{ Cichlidae (3) } \\
\hline $\begin{array}{l}\text { Cichlasoma orientale } \\
\text { Kullander,1983 }\end{array}$ & $\mathrm{F}$ & 41 & 8 & 5 & 0 & 6 & 8 & 68 & UFRN 1426 \\
\hline $\begin{array}{l}\text { Crenicichla menezesi Ploeg, } \\
1991\end{array}$ & $\mathrm{~F}$ & 11 & 12 & 0 & 0 & 0 & 6 & 29 & UFRN 1442 \\
\hline
\end{tabular}


Table 2. Continued...

\begin{tabular}{|c|c|c|c|c|c|c|c|c|c|}
\hline ORDER/Family/Species & FISIOLOGY & $\begin{array}{c}\text { MAIN } \\
\text { CHANNEL }\end{array}$ & STREAM & $\begin{array}{l}\text { FLOOD } \\
\text { PLAIN }\end{array}$ & $\begin{array}{l}\text { ROCK } \\
\text { POOL }\end{array}$ & POND & DAM & $\begin{array}{c}\text { TOTAL } \\
\text { ABUNDANCE } \\
\end{array}$ & VOUCHER \\
\hline $\begin{array}{l}\text { Oreochromis niloticus } \\
\text { (Linnaeus, 1758) }^{\text {(I) }}\end{array}$ & $\mathrm{F}$ & 23 & 3 & 0 & 0 & 8 & 12 & 46 & UFRN 2588 \\
\hline \multicolumn{10}{|l|}{ Eleotridae (2) } \\
\hline $\begin{array}{l}\text { Dormitator maculatus } \\
\text { (Bloch,1792) }\end{array}$ & $\mathrm{E}$ & 6 & 0 & 5 & 0 & 0 & 0 & 11 & UFRN 2613 \\
\hline Eleotris pisonis (Gmelin,1789) & $\mathrm{E}$ & 13 & 0 & 0 & 0 & 0 & 0 & 13 & UFRN 2577 \\
\hline \multicolumn{10}{|l|}{ Gobiidae (2) } \\
\hline $\begin{array}{l}\text { Awaous tajasica } \\
\text { (Lichtenstein, 1822) }\end{array}$ & $\mathrm{F}$ & 2 & 0 & 0 & 0 & 0 & 0 & 2 & $\begin{array}{l}\text { UFPB } \\
10028\end{array}$ \\
\hline $\begin{array}{l}\text { Gobionellus oceanicus } \\
\text { (Pallas, 1770) }\end{array}$ & M & 3 & 0 & 0 & 0 & 0 & 0 & 3 & UFRN 3415 \\
\hline \multicolumn{10}{|l|}{ PLEURONECTIFORMES (3) } \\
\hline \multicolumn{10}{|l|}{ Paralichthyidae (1) } \\
\hline \multicolumn{10}{|l|}{ Achiridae (2) } \\
\hline $\begin{array}{l}\text { Achirus achirus } \\
\text { (Linnaeus, } 1758 \text { ) }\end{array}$ & $\mathrm{E}$ & 3 & 0 & 0 & 0 & 0 & 0 & 3 & UFRN 3552 \\
\hline $\begin{array}{l}\text { Trinectes paulistanus (Miranda } \\
\text { Ribeiro, 1915) }\end{array}$ & $\mathrm{E}$ & 4 & 0 & 0 & 0 & 0 & 0 & 4 & $\begin{array}{l}\text { UFPB } \\
10030\end{array}$ \\
\hline \multicolumn{10}{|l|}{ TETRAODONTIFORMES (1) } \\
\hline \multicolumn{10}{|l|}{ Tetraodontidae (2) } \\
\hline $\begin{array}{l}\text { Lagocephalus laevigatus } \\
\text { (Linaeus, 1766) }\end{array}$ & M & 4 & 0 & 0 & 0 & 0 & 0 & 4 & UFRN 3544 \\
\hline $\begin{array}{l}\text { Sphoeroides testudineus } \\
\text { (Linnaeus, 1758) }\end{array}$ & $\mathrm{E}$ & 10 & 0 & 0 & 0 & 0 & 0 & 10 & UFRN 3543 \\
\hline $\begin{array}{l}\text { Total of species }(n=55) \\
\text { New record }(n=4)\end{array}$ & & 1,570 & 393 & 165 & 76 & 131 & 210 & 2,545 & \\
\hline
\end{tabular}

respectively. The most diverse families were Characidae, with 10 species, representing $18.2 \%$ of total, followed by Cichlidae and Poeciliidae with three species each (5.5\%) (Table 2). Of the five Cyprinodontiformes species listed, two belong to the family Cynolebiidae (Anablepsoides cearensis and Hypsolebias sp.). The non-annual killifish A. cearensis (Figure 2a and 2b) was the only threatened species in Mundaú river basin, listed as Critically Endangered (CR) in the Brazilian threatened fauna Red List (ICMBio 2014, Brasil 2014). This species was collected in two oxbow lakes (Figure 2d and 2e) and in a shallow perennial stream, located in an Arbustive Caatinga forest fragment (Figure $2 \mathrm{c}$ ), with similar features to the described type locality of the species (Costa \& Vono 2009).

\section{Discussion}

The predominance of the orders Characiformes and Siluriformes in the freshwater ichthyofauna of Mundaú river basin follows the pattern found among freshwater fishes in Brazilian Northeast (Ramos et al. 2005, Nascimento et al. 2014, Ramos et al. 2014, Silva et al. 2014), in Brazil (Buckup et al. 2007), and in Neotropical region (Reis et al. 2003, Lévêque et al. 2008). However, when estuarine and marine species are also considered, the order Perciformes is the most abundant, corroborating with other MNCE species listings (Soares-Filho et al. 2010, Paiva et al. 2014).

Astynax aff. bimaculatus was recorded in 54\% of sampling sites, being the most abundant species. It belongs to the Astyanax bimaculatus group composed by 22 valid species distributed along almost all drainages in South America, representing one of the most abundant morphotypes (Lucena \& Soares 2016). Together with Poecilia vivipara, Serrapinus heterodon, S. piaba, and Steindachnerina notonota, they represented the most common species and could exhibit small sized body, generalist ecophysiological habits, and partitioned or all year reproduction. These features, likewise others related to anthropic disturbances, such hypoxia and eutrophic habitats, are usually present in the most frequent and abundant species in freshwater community studies in MNCE (SánchezBotero et al. 2014, Silva et al. 2014). However, most of the species were restricted to a few habitats, reflecting narrow ecological conditions for their occurrence. The estuarine-marine fishes were mainly juveniles of species previously recorded in Mundaú Estuary, including some important ones for artisanal fisheries (Soares-Filho et al. 2010). Of the 25 non-freshwater species registered in the basin in our study, six are not mentioned in Soares-Filho et al. (2010): Citharichthys sp. Hemicaranx sp., Genyatremus luteus, Haemulopsis corvinaeformis, Larimus breviceps, and Sciades herzbergii. The same authors also observed the goliath grouper Epinephelus itajara (Lichtenstein, 1822) at Mundaú Estuary, a critically endangered marine species (Brasil 2014).

The occurrence of cynolebiids at temporary pools in the main channel and floodplains in the medium and lower ranges of Mundaú river basin may reinforce the biological importance of this drainage as a priority area for Caatinga conservation, as suggested by Brasil (2007) and in the recent reevaluation (Brasil 2016). Anablepsoides cearensis was only known from the type locality, a shallow stream in São Gonçalo do Amarante, Ceará (Costa \& Vono 2009). Thus, its distribution record was increased about $77 \mathrm{~km}$ west, to the lower portion of Mundaú river, in Itapipoca. Morphological differences between Hypsolebias sp. and its Caatinga congeners (Costa 2007) suggests that it may be a new species. The only species of the genus described for MNCE are: H. antenori (Tulipano, 1973), which occurs at the coastal basins east of Mundaú drainage, between Messejana municipality, in Ceará State, and Areia Branca municipality, in Rio Grande do Norte State (Costa 2007); H. longignatus Costa 2008 from Pacoti river basin, in Aquiraz municipality (Costa 2008), and H. martinsi from Icaraizinho river, in Amontada municipality (Britzke et al. 2016), 

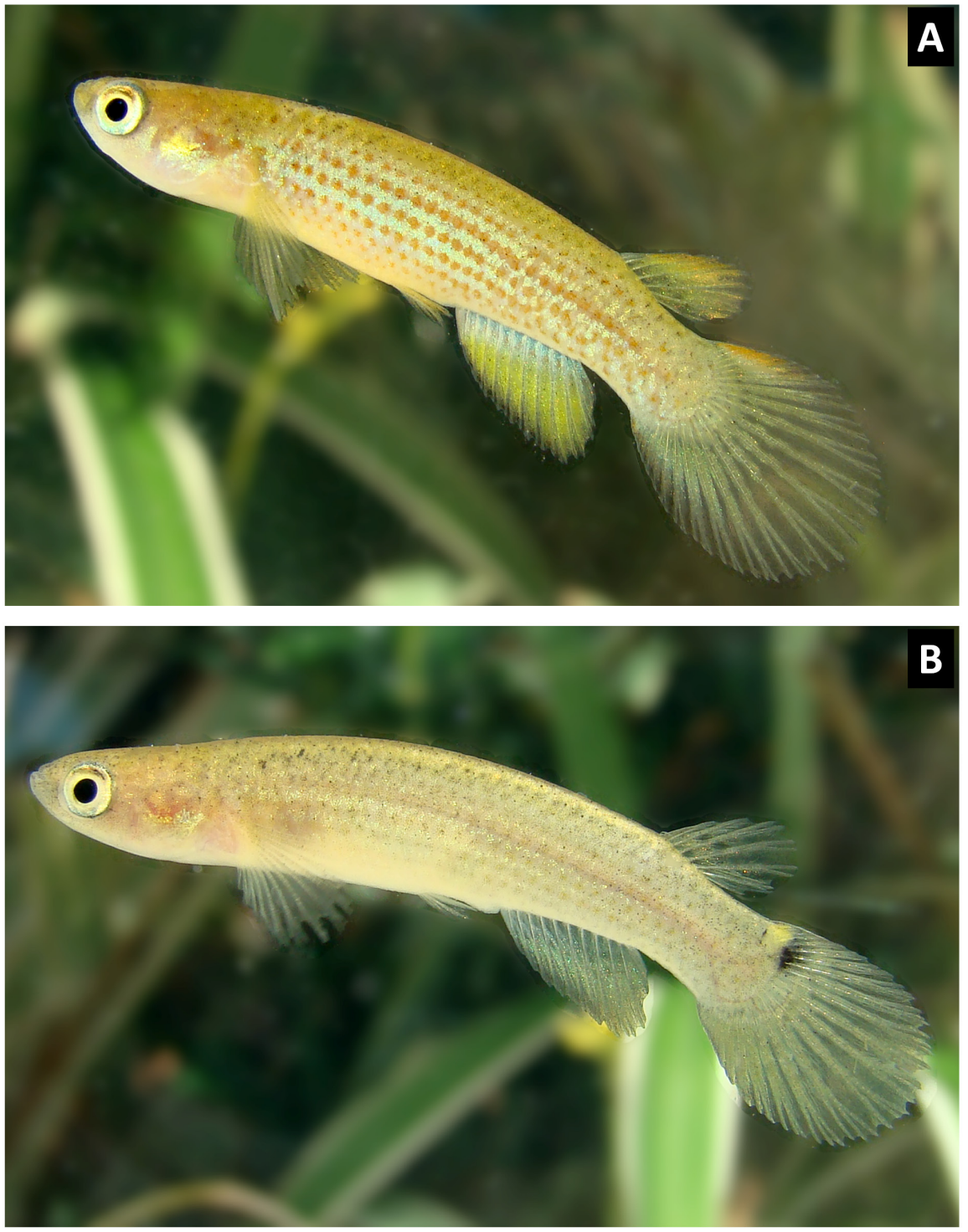
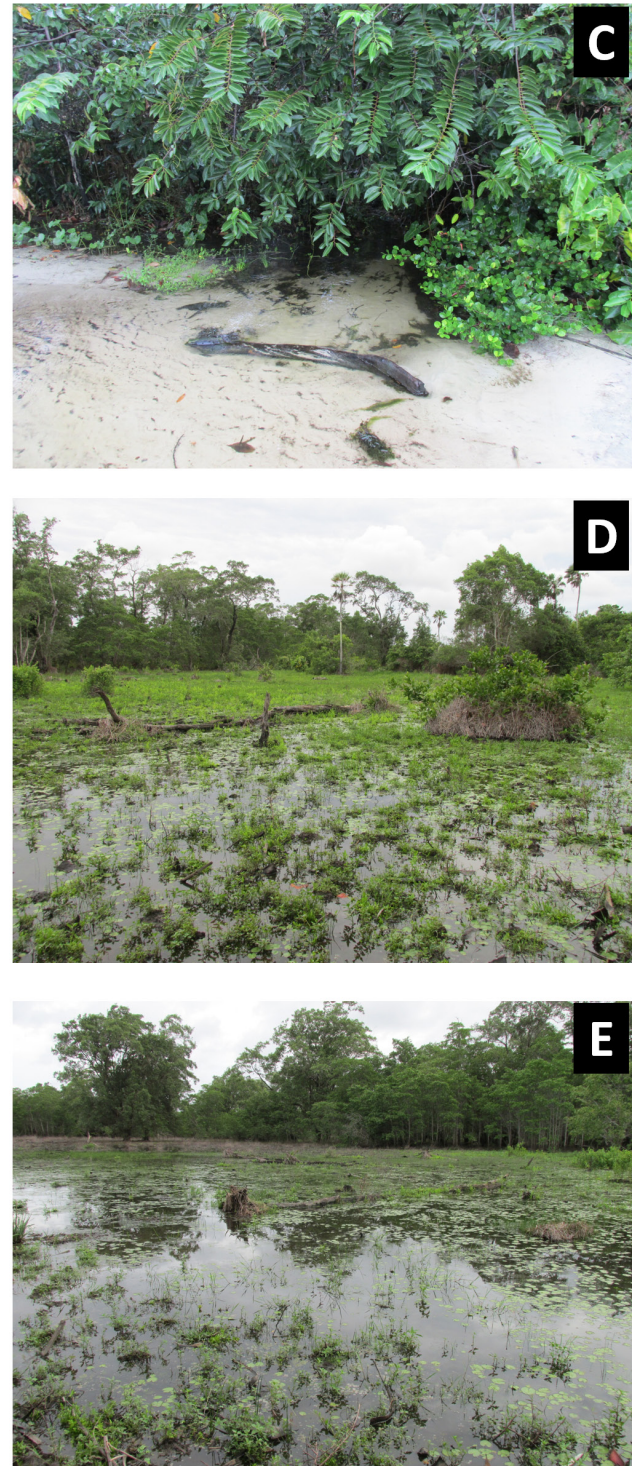

Figure 2. Anablepsoides cearensis, a critically endangered freshwater fish species collected in permanent pools in the lower portion of the Mundaú river basin. $\mathrm{A}=$ male, $\mathrm{B}=$ female, $\mathrm{C}, \mathrm{D}, \mathrm{E}=$ sampling sites.

both from small basins in Ceará State. Geographically, our record of Hypsolebias sp. is between the type locality of $H$. longignatus and $H$. martinsi, being the former eastern from Mundaú river basin and distant $140 \mathrm{~km}$, while the latter is on the northwest and closer, about $25 \mathrm{~km}$ in the adjacent Aracatiaçu river basin. However, Hypsolebias sp. seems to belong to the H. antenori group, and thus very distinct from $H$. longignatus, which belongs to the H. flammeus group. The species collected in Mundaú river basin differs from $H$. martinsi by the presence of vertical bars in body, and from $H$. antenori by the virtual absence of round blotches on caudal peduncle. Additional morphological and molecular studies should be done in order to determine its specific status. This record emphasizes the classification of MNCE as a freshwater ecoregion with a high percentage of endemic species (Albert et al. 2011).

Among the species labeled as new occurrences for MNCE, P. sarrafae was considered as endemic to Parnaíba river basin (Bragança \& Costa 2011, Ramos et al. 2014), and this is the first record outside Maranhão-Piauí ecoregion. Hemigrammus guyanensis was putatively endemic to Guiana
Shield, while H. rodwayi and Nannostomus beckfordi are considered as native from Amazon-Orinoco-Guianas Core (Albert et al. 2011) occurring in the Guiana, Suriname, French Guiana, and Northern Brazil (Reis et al. 2003, Buckup et al. 2007). H. rodwayi is also found in several MNCE coastal rivers: Mamanguape (UFPB 5685) and Abiaí (UFPB 9384) river basins, in Paraíba State, and Doce (UFRN 2483) and Jundiaí drainages (UFRN 2595), in Rio Grande do Norte State (unpublished data). The occurrence of those Amazonian species in Brazilian Northeast might be an indicative of a past connection between the Atlantic and Amazon forests in more humid periods (Wang et al. 2004).

Menezes et al. (2007) reported that Nannostomus beckfordi, recorded from Bahia and Alagoas states, was introduced in the Brazilian Northeast due to aquarism. However, the presence of this species in Mundaú river basin in Ceará State is the northern and westernmost record in MNCE, and it could be a relictual distribution and another evidence of the preterit connection between the Neotropical forested biomes. Currently, $N$. beckfordi is considered a poorly defined species complex, which needs a taxonomic 
review (Benzaquem et al. 2015). The native or introduced status of this taxon needs to be addressed once endemic undescribed species could be unprotected.

The introduction of Poecilia reticulata in Brazilian Northeast rivers can also be a result of aquarism. However, this species has been introduced in several countries for mosquito larvae control, such as Aedes aegypti (Lindholm et al. 2005). Meanwhile, larger species, such as tilapia Oreochromis niloticus, were introduced to fish farming in the reservoirs of the northeastern Brazil by the Departamento Nacional de Obras Contra Seca (DNOCS) in the 1970 (Paiva \& Mesquita 2013). In addition to competing for resources with native species, tilapias are quite resistant, easily surviving in disturbed environments (Leão et al. 2011).

A total of 55 species were found in Mundaú river basin, of which only two are introduced. The high species richness, together with new records for MNCE, which includes two cynolebiids, one of them a critically endangered species (Anablepsoides cearensis), highlights the importance of preserving this basin, mainly the lowland stretches. However, some anthropic impacts were observed along Mundaú river basin waterbodies, mainly associated to riparian forest removal for agriculture and cattle raising in many stretches of the rivers and streams sampled; river sand extraction; damming; irregular occupation of river margins by human habitation; and domestic sewage discharge. The ichthyological survey of Mundaú river basin contributes to the knowledge of Caatinga fish fauna, and corroborates its importance as a priority area for the conservation of aquatic biota of this semiarid region.

\section{Acknowledgements}

We thank Conceição Teixeira, Miguel Pires, Elissângela Teixeira, Chagas Sousa, Evaldo Carlos, Tiago Sousa, André Pereira, Victor Távora, Frederico Garcia and Luana Magalhães for helping during fieldwork. Roberto Feitosa, Bianca Terra, Cynthia Ogawa, José Louvise and Márcio Silva for contributing with laboratory activities. Keilo Teixeira was funded by FUNCAP (Fundação Cearense de Apoio ao Desenvolvimento Científico e Tecnológico), and Project was partially funded by CAPES (AUXPE 23038.005584/2012-20).

\section{References}

ALBERT, J.S., PETRY, P. \& REIS, R.E. 2011. Major biogeographic and phylogenetic patterns. In Historical Biogeography of Neotropical Freshwater Fishes (J.S. Albert \& R.E. Reis, eds). Berkeley University of California Press, p.21-58.

ARAÚJO, M.E., TEIXEIRA, J.M.C. \& OLIVEIRA, A.M.E. 2004. Peixes estuarinos marinhos do Nordeste do Brasil: guia ilustrado. Edições UFC, Fortaleza.

BRAGANÇA, P.H.N. \& COSTA, W.J.E.M. 2011. Poecilia sarrafae, a new poeciliid from the Parnaíba and Mearim river basins, northeastern Brazil (Cyprinodontiformes: Cyprinodontoidei). Ichthyol. Explor. Fresh. 21(4): 369-376.

BENZAQUEM, D.C., OLIVEIRA, C., BATISTA, J.S., ZUANON, J. \& PORTO, J.I.R. 2015. DNA Barcoding in Pencilfishes (Lebiasinidae: Nannostomus) Reveals Cryptic Diversity across the Brazilian Amazon. PLoS ONE 10(2): 1-14.

BRASIL. 2007. Ministério do Meio Ambiente. Áreas Prioritárias para a Conservação, Uso Sustentável e Repartição de Benefícios da Biodiversidade Brasileira: Atualização - Portaria MMAN 9, de 23 janeiro de 2007. Brasília: MMA. p.300.

BRASIL. 2014. Lista Nacional Oficial de Espécies da Fauna Ameaçada de Extinção - Portaria $N^{\circ} 443$, de 17 de dezembro de 2014. Ministério do Meio Ambiente. Diário Oficial da União, Brasília, Seção 1(245): 121-130.

BRASIL. 2016. Ministérios do Meio Ambiente. Resultados da $2^{\mathrm{a}}$ atualização das Áreas e Ações Prioritárias para Conservação, Uso Sustentável e Repartição dos Benefícios da Biodiversidade dos biomas Cerrado e Pantanal realizado em 2012, e da Caatinga, realizado em 2015. http://www.mma.gov.br/biodiversidade/ biodiversidade-brasileira/\%C3\%A1 reas-priorit $\% \mathrm{C} 3 \% \mathrm{~A} 1$ rias/item $/ 10724$ (the last access in 13/09/2016).
BRITSKI, H.A., SATO, Y. \& ROSA, A.B.S. 1984. Manual de Identificação de Peixes da Região de Três Marias: Com Chave de Identificação Para os Peixes da Bacia do São Francisco. Brasília: Câmara dos Deputados, Coordenação de Publicações - CODEVASF, Divisão de Piscicultura e Pesca

BRITZKE R., NIELSEN, D.T.B. \& OLIVEIRA, C. 2016. Description of two new species of annual fishes of the Hypsolebias antenori species group (Cyprinodontiformes: Rivulidae), from Northeast Brazil. Zootaxa 4114(2):123-138.

BUCKUP, P.A., MENEZES, N.A. \& GHAZZI, M.S. 2007. Catálogo das Espécies de Peixes de Água Doce do Brasil. Rio de Janeiro, Museu Nacional, p. 1-195.

COGERH. 2014. Companhia de Gestão dos Recursos Hídricos. Bacias hidrográficas do Litoral Características Gerais. http://portal.cogerh.com.br/downloads/ category/83-pacto-das-aguas-plano-estrategico (the last access in 02/11/2014)

COSTA, W.J.E.M. 2007. Taxonomic revision of the seasonal South American killifish genus Simpsonichthys (Teleostei: Cyprinodontiformes: Aplocheiloidei: Rivulidae). Zootaxa. (1669): 1-134.

COSTA, W.J.E.M. 2008. Simpsonichthys longignatus, a new seasonal killifish of the S. flammeus group from the Pacoti river basin, northeastern Brazil (Cyprinodontiformes:Rivulidae). Ichthyol. Explor. Fresh. 19(2): 155-159.

COSTA, W.J.E.M. \& VONO, V. 2009. Rivulus cearensis, a new aplocheiloid killifish from northeastern Brazil (Cyprinodontiformes:Rivulidae). Ichthyol Explor. Fresh. 20(1): 7-11

ESCHMEYER, W.N. 2015. Catalog of fishes: genera, species, references. http:// research.calacademy.org/research/ichthyology/catalog/fishcatmain.asp (the last access in 06/04/2015)

FISHBASE. http://www.fishbase.org/home.htm (the last access in 09/10/2014)

FUNCEME. 2015. Fundação Cearense de Meteorologia e Recursos Hídricos. http:// www.funceme.br/app/calendario/produto/municipios/maxima/diario?data $=$ hoje (the last access in 24/03/2015)

GOMES-FILHO, G. \& ROSA R.S. 2001. Inventário da Ictiofauna da Bacia do Rio Gramame, Paraíba, Brasil. In A Bacia do Rio Gramame: Biodiversidade, Uso e Conservação (T. Watanabe, ed.). PRODEMA, João Pessoa, p.167-173.

GURGEL-LOURENÇO, R.C., SOUSA, W.A., SÁNCHEZ-BOTERO, J.I. \& GARCEZ, D.S. 2013. Ichthyofauna of two reservoirs in the middle Acaraú river. Check List 9(6): 1391-1395.

IPECE. 2012. Instituto de Pesquisa e Estratégia Econômica do Ceará. http://www. ipece.ce.gov.br/publicacoes/perfil_basico/pbm-2013/Itapipoca.pdf (the last access in 01/01/2015)

JEREP, FC \& MALABARBA, L.R. 2014. A new species of Serrapinnus Malabarba, 1998 (Characidae: Cheirodontinae) from Rio Grande do Norte State, northeastern Brazil. Neotrop. Ichthyol. 12(2): 301-308.

KULLANDER, S.O. 1988. A revision of the South American cichlid genus Cichlasoma (Teleostei: Ciclidae). Swedish Museum of Natural History, Stockholm.

LANGEANI, F., BUCKUP, P.A., MALABARBA, L.R., PY-DANIEL, L.H.R., LUCENA, C.A.S., ROSA, R.S., ZUANON, J.A.S., LUCENA, Z.M.S., BRITTO, M.R., OYAKAWA, O.T. \& GOMES-FILHO, G. 2009. Peixes de água doce. In Estado da arte e perspectivas para a zoologia no Brasil (R.M. Rocha \& W.A. Boeger, eds). Sociedade Brasileira de Zoologia, Volume I, Curitiba, p.211-230.

LEÃO, T. C. C., ALMEIDA, W.R., DECHOUM, M.S., ZILER, S.R. 2011. Espécies exóticas invasoras no Nordeste do Brasil: contextualização, manejo e políticas públicas. Recife: Centro de Pesquisas Ambientais do Nordeste e Instituto Hórus de Desenvolvimento e Conservação Ambiental. 99 p.

LÉVÊQUE, C., OBERDORFF, T., PAUGY, D., STIASSNY, M.L.J., \& TEDESCO, P.A. 2008. Global diversity of fish (Pisces) in freshwater. Hydrobiologia. 595(1): 545-567.

LINDHOLM, A.K., BREDEN, F., ALEXANDER, H.J., CHAN, W.K., THAKURTA S.G., \& BROOKS, R. 2005. Invasion success and genetic diversity of introduced populations of guppies Poecilia reticulata in Australia. Mol. Ecol. 14(12): 3671-3682.

LUCENA, C.A.S. \& SOARES, H.G. 2016. Review of species of the Astyanax bimaculatus "caudal peduncle spot" subgroup sensu Garutti \& Langeani (Characiformes, Characidae) from the rio La Plata and rio São Francisco drainages and coastal systems of southern Brazil and Uruguay. Zootaxa 4072 (1): $101-125$. 
LUCENA, C.A. S., CALEGARI, B.B., PEREIRA, E.H.L. \& DALlEGRAVE, E. 2013. O uso de óleo de cravo na eutanásia de peixes. Boletim Sociedade Brasileira de Ictiologia 105: 20-24.

MARCENIUK, A. P. Chave para identificação das espécies de bagres marinhos (siluriformes, ariidae) da costa brasileira. Boletim do Instituto de Pesca, São Paulo 31(2): $89-101$.

MENEZES, N.A., WEITZMAN, S.H., OYAKAWA, O.T., LIMA, F.C.T., CASTRO, R.M.C., \& WEITZMAN, M.J. 2007. Peixes de água doce da Mata Atlântica: lista preliminar das espécies e comentários sobre conservação de peixes de água doce neotropicais. Museu de Zoologia da Universidade de São Paulo, São Paulo.

NASCIMENTO, W.S., BARROS, N.H.C., ARAÚJO, A.S., GURGEL, L.L., CANAN, B., MOLINA, W.F., ROSA, R.S. \& CHELLAPPA, S. 2014. Composição da ictiofauna das bacias hidrográficas do Rio Grande do Norte, Brasil. Bio. Amaz. 4(1): 126-131.

PAIVA, M.P. \& MESQUITA, P.E.C. 2013. Uma semente fecunda: Commissão Téchnica de Piscicultura do Nordeste $(1932$ - 1945). Revista do Instituto do Ceará. p.10-40.

PAIVA, R.E.C., LIMA, S.M.Q., RAMOS, T.P.A. \& MENDES L.F. 2014. Fish fauna of Pratagi river coastal microbasin, extreme north Atlantic Forest, Rio Grande do Norte State, northeastern Brazil. Check List 10(5): 968-975.

PLOEG, A. 1991. Revision of the South American cichlid genus Crenicichla Heckel, 1840 , with descriptions of fifteen new species and considerations on species groups, phylogeny and biogeography. Academisch Proefschrift, Universiteit van Amsterdam, Amsterdam.

RAMOS, R.T.C., RAMOS, T.P.A., ROSA, R.S., BELTRÃO, G.B.M. \& GROTH, F. 2005. Diversidade de Peixes (Ictiofauna) da bacia do rio Curimataú, Paraíba. In Análise das Variações da Biodiversidade do Bioma Caatinga: Suporte das Estratégias Regionais de Conservação (F.S. Araujo, M.J.N. Rodal \& M.R.V. Barbosa, eds.), Ministério do Meio Ambiente, Brasília, p.291-318.

RAMOS, T.P.A., R.T.C. RAMOS and S.A.Q.A. RAMOS. 2014. Ichthyofauna of the Parnaíba river Basin, Northeastern Brazil. Biota Neotrop. 14(1): 1-8. www. biotaneotropica.org.br/v14n1/en/abstract?inventory+bn01014012014 (the last access in 17/02/2016).

RAMOS, T.P.A. 2012. Ictiofauna de água doce da bacia do rio Parnaíba. Tese de doutorado, Universidade Federal da Paraíba, João Pessoa.
REIS, R.E., KULLANDER, S.O. \& FERRARIS JR, C.J. 2003. Check list of the freshwater fishes of South Central and America, Porto Alegre.

ROSA, R.S. \& GROTH F. 2004. Ictiofauna dos Ecossistemas de Brejos de Altitude de Pernambuco e Paraíba. In Brejos de Altitude em Pernambuco e Paraíba: História Natural, Ecologia e Conservação (K.C. Pôrto, J.J.P. Cabral \& M. Tabarelli, orgs.). Série Biodiversidade, Ministério do Meio Ambiente, Brasília, p.201-210.

ROSA, R.S., MENEZES, N.A., BRITSKI, H.A., COSTA, W.J.E. \& GROTH F. 2003. Diversidade, padrões de distribuição e conservação dos peixes da caatinga. In Ecologia e Conservação da Caatinga (I.R. Leal, M. Tabarelli \& J.M.C. Silva eds.). Editora Universitária da Universidade Federal de Pernambuco, Recife, p.135-180.

SÁNCHEZ-BOTERO, J. I., REIS, V.C., CHAVES, F.D.N. \& GARCEZ, D.S. 2014. Fish assemblage of the Santo Anastácio reservoir (Ceará State, Brazil). Boletim do Instituto de Pesca. 40(1): 1-15.

SEMACE. 2014. Superintendência Estadual do Meio Ambiente - Área de Proteção Ambiental do Estuário do Rio Mundaú. http://www.semace.ce.gov.br/2010/12/ area-de-protecao-ambiental-do-estuario-do-rio-mundau/ (the last access in 07/10/2014)

SILVA, M.J., RAMOS, T.P.A., DINIZ, V.D., RAMOS, R.T.C. \& MEDEIROS, E.S.F. 2014. Ichthyofauna of Seridó/Borborema: a semi-arid region of Brazil. Biota Neotrop. 14(3): 1-6. http://dx.doi.org/10.1590/1676-06032014007713 (the last access in 17/02/2016)

SOARES FILHO, A.A., SAMPAIO, C.M.S., FERREIRA, T.D., LOURENÇO, J.A., SOUSA, R.A.L. \& IGARASHI M.A. 2010. Contribuição ao conhecimento da Ictiofauna estuarina no Estado do Ceará, Brasil. Revista da Gestão Costeira Integrada 8: 1-9.

WANG, X., AUGUSTO, S.A., EDUARDS, R.L., CHENG, H., CRISTALLI, P.S., SMART., P.L., RICHARDS, D.A. \& SHEN, C.C. 2004. Wet periods in northeastern Brazil over the past $210 \mathrm{kyr}$ linked to distant climate anomalies. Nature 432: 740-743.

Received: 18/02/2016

Revised: $02 / 11 / 2016$

Accepted: 07/12/2016

Published online: 16/01/2017 